\title{
Tolerance of Ambiguity: A Review of the Recent Literature
}

\author{
Adrian Furnham ${ }^{1,2}$, Joseph Marks ${ }^{3}$ \\ ${ }^{1}$ Research Department of Clinical, Educational and Health Psychology, \\ University College London, London, UK \\ ${ }^{2}$ Norwegian Business School, Olso, Norway \\ ${ }^{3}$ School of Psychology, University of Birmingham, Birmingham, UK \\ Email: a.furnham@ucl.ac.uk
}

Received July $10^{\text {th }}, 2013$; revised August $8^{\text {th }}, 2013$; accepted August $31^{\text {st }}, 2013$

\begin{abstract}
Copyright (C 2013 Adrian Furnham, Joseph Marks. This is an open access article distributed under the Creative Commons Attribution License, which permits unrestricted use, distribution, and reproduction in any medium, provided the original work is properly cited.
\end{abstract}

\begin{abstract}
This review paper attempts to update the literature on tolerance of ambiguity (TA) and related concepts since a previous review (Furnham \& Ribchester, 1995). Various related concepts like Uncertainly Avoidance and In/Tolerance of Uncertainly are reviewed. Both correlational and experimental studies of TA are reviewed and tabulated. Further, an attempt was made to identify and critique various different questionnaires design to measure TA. Recommendations for the use of these tests in research are made. The reasons for progress and lack of progress in this field are highlighted.
\end{abstract}

Keywords: Tolerance; Ambiguity; Review

\section{Introduction}

The concept of tolerance of ambiguity (TA), which was originally developed by Frenkel-Brunswik (1948), has attracted a great deal of research over the last 60 years (Merrotsy, 2013). Her paper, that related TA to authoritarianism, has since been cited nearly 10,000 times (Adorno, Frenkel-Brunswik, Levinson, \& Sanford, 1950). TA has generally been conceived as a personality variable or individual difference factor (Budner, 1962) and has been used in a variety of applied fields, including clinical psychology (Lachance et al., 1999), medicine (Geller et al., 1993) and organisational behaviour (Judge et al. 1999). This paper attempts to update review by Furnham (1994) and Furnham and Ribchester (1995) on the conceptions, correlates and measurement of TA.

\section{History of the Concept}

Frenkel-Brunswick (1949) defined TA as an "emotional and perceptual personality variable". She was influenced by the work of Jaensch (1938) whose work was to influence many others (Eysenck, 1954). She concluded: "In the present paper, an attempt was made to discuss denial of emotional ambivalence and intolerance of cognitive ambiguity as but different aspects of what may be a fairly coherent characteristic. An underlying emotional conflict between glorification and hostility in the attitude towards parents, sex and one's own social identity previously demonstrated in children inclined toward rigid social dichotomizing as revealed by ethnic prejudice is taken as the impetus for experiments in memory, perception, and related topics, devised to test tolerance of ambiguity on an emotionally more neutral ground. There is some indication of a prevalence of premature reduction of ambiguous cognitive patterns to certainty in the prejudiced subjects, as revealed by a clinging to the familiar, or by a superimposition of one or many distorting cliches upon stimuli which are more manageable in a more simple and stereotyped fashion. There is some indication that in the case of distinct intolerance of emotional ambivalence one may as a rule be able to locate at least some aspects of intolerance of cognitive ambiguity although these may often by more apparent on a higher level than that of perception paper." (p. 140).

Frenkel-Brunswick (1951) set out many behavioural features of TA including resistance to reversal of apparent fluctuating stimuli; the early selection and maintenance of one solution in a perceptually ambiguous situation; inability to allow for the possibility of good and bad traits in the same person; acceptance of attitude statements representing a rigid; black-white view of life; seeking for certainty; a rigid dichotomising into fixed categories; premature closure, and remaining closed except to familiar characteristics of stimuli. Thus TA was conceived as a salient, multi-faceted predictive variable in a variety of behavioural settings.

Frenkel-Brunswick's $(1949,1951)$ definition of the concept was generated by case study material gleaned from interviews of persons high or low on this construct. She argued that TA generalises to the various aspects of emotional and cognitive functioning of the individual, characterising cognitive style, belief and attitude systems, interpersonal and social functioning and problem solving behaviour. She also related TA to other personality variables, predicting a positive relationship with the authoritarian family of personality traits. Since then the topic has attracted considerable research and remains a well-used variable to this day (Anderson \& Schwartz, 1992; Merrotsy, 2013).

\section{Early Studies}

Many of the early studies in this area were psychometric studies that attempted to construct a valid, self-report, measure 
of TA. Budner (1962) defined TA as "the tendency to perceive ambiguous situations as desirable" and set about one of the first measures in the field. Budner's (1962) paper has been cited over 1000 times. McLain (1993) included contextual information, defining TA as "a range, from rejection to attraction, of reactions to stimuli perceived as unfamiliar, complex, dynamically uncertain or subject to multiple conflicting interpretations" (p. 184).

There has expectedly been debate on the dimensionality of the TA concept. Durrheim and Foster (1997) propose that TA is a context-specific construct, not a personality trait, and others advocate the use of contextualised measures (Herman, Stevens, Bird, Mendenhall, \& Oddou, 2010).

TA is however usually measured on a one-dimensional scale: those who are intolerant of ambiguity are described as having a tendency to resort to black-and-white solutions, and characterised by rapid and overconfident judgement, often at the neglect of reality (Frenkel-Brunswik, 1949). At the other end of the scale, ambiguous situations are perceived as desirable, challenging and interesting, usually by individuals who score highly on an Openness to Experience scale (Caligiuri, Jacobs, \& Farr, 2000) and show both sensation-seeking and risk-taking behaviour (McLain, 1993; Lauriola, Levin, \& Hart, 2007; McLain, 2009).

Generally for those with low TA there is an aversive reaction to ambiguous situations because the lack of information makes it difficult to assess risk and correctly make a decision. These situations are perceived as a threat and source of discomfort. Reactions to the perceived threat are stress, avoidance, delay, suppression, or denial (Budner, 1962; MacDonald, 1970; McLain, 1993; Furnham \& Ribchester, 1995).

In more recent work researchers have altered their focus towards examining how TA influences the perception of situations and decision making (Yurtsever, 2001, 2008; Van Hook \& Steele, 2002; McLain, 2009). Instead it is thought that constructs that are defined by an absence of information (e.g. risk-taking behaviour) are more relevant and are more useful validating TA measures.

The TA research literature appears to have three different features: there have been work on similar concepts to TA which is discussed below; there have been an increase of experimental over correlational studies; and a more of an interest in the effects of TA in the work environment.

\section{Conceptual and Definitional Issues}

Other similar concepts have been described which are clearly very similar to TA like Uncertainty Avoidance and Risk-Taking Propensity. Hofstede (1984) recognised uncertainty avoidance as a characteristic of cultures and developed an uncertainty avoidance index. The distribution of the personality variable "uncertainty avoidance" has been studied in different societies, thus making it a "sociological variable", where uncertainty avoidance was defined as "the extent to which people feel threatened by ambiguous situations, and have created beliefs and institutions that try to avoid these" (p. 419). Most researchers interested in uncertainty avoidance are cross-cultural or organisational psychologists who are interested in comparing groups of individuals, rather than seeing it as an individual preference or trait. As a consequence, these researchers seem not to have developed many self-report measures of uncertainty avoidance.
There is also a clinical literature on Tolerance of Uncertainty (TU) which has been conceived of as a cognitive disposition that confers risk of Generalised Anxiety Disorder (Birrell, Meares, Wilkinson \& Freeston, 2011). Various self-report measures have been developed which are often validated against measures of anxiety, depression and worry (Carleton, Norton \& Asmundson, 2007). TU is associated with worries and negative expectations of the future and is therefore often involved in research of anxiety disorders (Ladouceur et al., 2000). TU is usually measured using the Intolerance of Uncertainty Scale (Freeston et al., 1994), which is made up of 27 items. Its internal consistency is high, $\alpha=0.91$ and Dugas et al. (1997) reported a test-retest reliability of 0.78 over a five week period. The scale is used as a clinical tool in the diagnosis of GAD (Freeston et al., 1994). It continues to be examined for its psychometric properties (Buhr \& Dugas, 2002; Fergus \& Wu, 2012).

Green and Roger (2001) argued that there is a clear relationship between TA and TU but that the former is used primarily in cognitive studies on decision-making, memory and perception "all of which are oriented towards cognitive processes rather than stress and emotion" (p. 521). They developed a three factor scale two factors of which (emotional uncertainty and cognitive uncertainty) were modestly correlated (respectively: $r=.18$ and $r=.37, N=204$ ). In their review of the factor analytic studies of the best known scale in the area, Birrell et al (2011) found evidence of two factors: Desire for Predictability and Active Engagement in Seeking Certainty; and Paralysis of Cognition and Cognition in the Face of Uncertainty.

The TA, TU and uncertainty avoidance concepts have been used interchangeably (Stewart, Carland, Carland, Watson, \& Sweo, 2003; McLain, 1993; Majid \& Pragasam, 1997; Grenier, Barrette, \& Ladouceur, 2005) but efforts have been made to show that the concepts are not identical. Ellsberg (1961) defined ambiguity as a lack of information that is necessary to understand a situation or to identify all of the possible outcomes. Krohne $(1989,1993)$ concluded that whilst ambiguity is a property of the stimulus, uncertainty is the emotional state that is provoked by it. Grenier et al. (2005) argued that the main difference between TA and TU is the time frame referred to. TA describes a trait that focuses on an individual's reaction to an ambiguous situation in the present. TU, on the other hand, describes a future-orientated trait, where the individual is reacting to the uncertainty of the future. This discrimination partly explains why the TA and TU literature tend to have separate areas of focus. TA is used in the cognitive and experimental literature and TU in the clinical literature.

Risk-taking propensity is also very similar to TA (McLain, 2009). Lauriola, Levin and Hart (2007) argue that there is a stable dispositional trait that underlies risky decision making and decision making under ambiguity in experimental tasks. Ellsberg (1961) distinguished decisions under ambiguity from risky decision making in terms of knowledge of outcomes and probabilities: Behavioural decision scientists usually define ambiguous decision making as a situation in which there is an unknown distribution of outcome probabilities for at least one of the options. Whereas the probabilities are known in risky decision-making, but outcomes are not.

However it remains true that despite work on these subtly different and related concepts there is still no very clear operational definition of TA at the facet level or a clear differentiation between the manifestations and correlates of TA. Nor has 
there been any strong theoretical development in the area. Both may account for the steady, but uninspiring, developments in the area.

\section{Correlational Studies}

There have been many attempts to look at the concurrent, convergent and discriminant validity of TA with studies correlating scores with other established measures. Most of the work in this area remains correlational.

The relationships between TA and other personality variables have been supported by correlations in self-report questionnaires and has been used to validate scales of TA. Budner's (1962) 16-item scale positively correlated with authoritarianism and this was used as evidence of construct validity. MacDonald's (1970) AT-20 correlated with Rokeach's dogmatism scale and the Walk's A Scale correlated with ethnocentrism (O'Connor, 1952).

Correlations also exist with other forms of measurement of TA. Million (1957) measure TA by the autokinetic phenomenon and found a relationship with authoritarianism. However despite some evidence for this relationship, research has not always proved conclusive. For example, Feather (1969) found that the Budner scale's measurement of TA did not relate to dogmatism.

We set about an extensive search for all TA and TU papers published since 1995. Many simply mentioned the concepts, and we decided to review only those which had actually used a measure of TA or TU in the research. We then decided to tabulate the results showing the range of papers published, what measures they used and what they found. These are shown in Table 1 where 30 studies are reviewed.

Because details of the studies are provided in the table the results will not be considered in detail. Rather, four observations from this research effort can be summarised. First, they use a wide range of measures of TA, not all of which correlate very highly with each other. Second, many have modest population groups, though a number have populations over 200 . Third, the number of variables correlated with measures of TA were extremely varied from art preference, though identity conflict to thinking style. There seemed no thematic or programmatic effort on any research group in this area. Correlations tended to be modest. Fourth, most studies had their hypotheses confirmed showing how TA was conceptually related to a variety of other measures and behaviours.

\section{Experimental Work}

There have also been one or two experimental studies in this area. However they have been the exception rather than the rule. For instance Lauriola and Levin (2001) designed an ecologically valid experiment that compared attitudes towards ambiguity and risk. They showed that differences in attitude towards ambiguity are consistent with attitudes towards risk, in that a preference for the ambiguous predicts a preference for a risky options. However on further inspection, the relationship only proved significant when participants were avoiding a loss in the Risky Decision-making Task as opposed to seeking a gain. It was concluded that the extra processing in the "loss" condition meant that attitudes towards ambiguity were more important in the decision-making procedure.

Lauriola, Levin and Hart (2007) repeated this experiment and found that the Ambiguity-Probability Tradeoff task negatively correlated with a TA self-report questionnaire (MSTAT-I) $(\mathrm{r}=$ $-.15 ; p<0.05)$ as well as optimism scores on the Life Orientation Test-Revised, and positively correlated with regret-based decision style. A high score on this task predicted subsequent risky choices in a follow up study a month later. It was also predictive of later ambiguous choices in a different domain. These findings support the existence of a stable dispositional trait underlying reactions to risk and ambiguity.

\section{The Measurement of TA}

Given that the TA construct has been around for so long, it is no surprise that a number of measures exist (see Table 2). To date we have found 8 self-report measures. However we acknowledge other, unpublished, tests are recorded (Saunders, 1955) or those where little psychometric work was attempted (Eysenck, 1954). Eysenck's early measure was a simple 14item true-false test but appeared to have good concurrent validity. He reported on a non-verbal pictorial intolerance of ambiguity test which was 8 drawings of a dog turning slowly and by degrees into a cat. The predicted and confirmed hypothesis was that rigid people would continue to cling to the original "dog" concept long after it had turned into a cat.

Most of the tests of TA are however self-report questionnaires. One of the best known and well used scales in this area was developed 30 years ago by Budner (1962) who devised a 16-item (half positive, half negative) scale which was described in great detail. He argued that each item had to tap at least one postulated indicator of perceived threat, namely phenomenological submissions or denial, operative submission or denial. Items referred to one of either of three types of ambiguous situations: novelty, complexity and insolubility. The scale was validated on 17 different, mainly student, populations and shown to be free of acquiescent and social desirability response tendencies. Although the test correlation was good ( 0.85 over 2 months) the internal alpha was poor (0.49). Various forms of validity were examined including concurrent and construct.

Budner's scale was correlated with rankings of individuals on the basis of short biographies, peer ratings, and measures of conventionality, belief in divine power, attendence at religious services, dogmatism about religious beliefs and attitudes to censorship. The total scale also correlated positively with authoritarianism, idealism of and submission to parents, Machiavellianism, career choice in medical students etc. Not all the correlations were significant and most were in the 0.20 to 0.40 range but they were sufficiently consistent to suggest that the measure had content, concurrent and construct validity. The wording of items in this scale have been criticised for their failure to represent the appropriate stimulus, or even suggest ambiguity at all (McLain, 2009). The items are also argued to be confounded by reference to specific situation, which may elicit misleading reactions. Budner saw TA as a "non-specific" trait that does not lead to specific behaviours or evaluations that are not manifestations of TA itself.

The Budner scale has perhaps attracted most attention and is used most frequently in TA research. Rydell and Rosen (1966) and Rydell (1966) reported on the development and validation of another scale. The scale consisted of 16 true-false items which had been constructed on a "a-priori basis" (Rydell \& Rosen, 1966: p. 151) with relatively limited validation. Testretest reliabilities over a month with 41 students yielded an $r=$ 


\section{A. FURNHAM, J. MARKS}

Table 1.

Table showing TA measures.

\begin{tabular}{|c|c|c|c|c|c|}
\hline Authors & $\mathbf{N}$ & TA measure & Outcome measure & $\mathbf{r} / \boldsymbol{p}$ values & Findings \\
\hline Thalbourne et al. (2000) & 100 & AT-20 & Transliminality & $\mathrm{r}=-0.02$ & Transliminality did not correlate with TA. \\
\hline Litman, (2010) & 372 & AT-20 & $\begin{array}{l}\text { Dispositional interest (I)-type } \\
\text { curiosity; Deprivation (D)-type } \\
\text { curiosity; Anxiety; Anger; } \\
\text { Depression }\end{array}$ & $\begin{array}{l}r=0.36 ;-0.15 \\
-0.02 ; 0.03 ;-0.15\end{array}$ & $\begin{array}{l}\text { AT- } 20 \text { scale was positively correlated with I-type } \\
\text { curiosity and negatively correlated to D-type } \\
\text { curiosity and anger. }\end{array}$ \\
\hline Weisbrod, (2009) & 157 & AT-20 & Ethical decision making & $p=0.018,<0.01$ & $\begin{array}{l}\text { Low TA predicts less willingness to violate } \\
\text { ethical norms in both personal and organizational } \\
\text { settings. High TA subjects were more likely to } \\
\text { violate ethical norms whilst experiencing high } \\
\text { negative affect. }\end{array}$ \\
\hline $\begin{array}{l}\text { Hazen, Overstreet, } \\
\text { Jones-Farmer, Field, } \\
\text { (2012) }\end{array}$ & 340 & $\begin{array}{l}\text { Multiple Stimulus } \\
\text { Types Ambiguity } \\
\text { Tolerance Scale-II } \\
\text { (MSTAT-II) }\end{array}$ & $\begin{array}{l}\text { Willingness to pay for } \\
\text { remanufactured goods }\end{array}$ & $p<0.001 ; p<0.001$ & $\begin{array}{l}\text { Consumers' TA positively correlated with their } \\
\text { willingness to pay for remanufactured products; } \\
\text { TA positively correlated with perceived quality } \\
\text { of remanufactured products. }\end{array}$ \\
\hline \multirow[t]{5}{*}{$\begin{array}{l}\text { Ramdeny, } \\
\text { (2009) }\end{array}$} & 510 & $\begin{array}{c}\text { Uncertainty } \\
\text { Tolerance Scale } \\
\text { (UTS; Dalbert, 1999) }\end{array}$ & Challenge appraisal & $\mathrm{r}=-0.12, p<0.05$ & $\begin{array}{l}\text { Intolerance of ambiguity negatively correlated } \\
\text { with challenge appraisal. }\end{array}$ \\
\hline & & & Life satisfaction & $\begin{array}{c}\mathrm{r}=-0.14, p<0.05 \\
\mathrm{r}=-0.15, p<0.05\end{array}$ & $\begin{array}{l}\text { Intolerance of ambiguity negatively correlated } \\
\text { with life satisfaction. }\end{array}$ \\
\hline & & & Positive affect & $\begin{array}{c}\mathrm{r}=-0.24, p<0.01 \\
\mathrm{r}=-0.18, p<0.01\end{array}$ & $\begin{array}{l}\text { Intolerance of ambiguity negatively correlated } \\
\text { with positive affect. }\end{array}$ \\
\hline & & & Threat appraisal & $\mathrm{r}=0.30, p<0.01$ & $\begin{array}{l}\text { Intolerance of ambiguity positively correlated } \\
\text { with threat appraisal. }\end{array}$ \\
\hline & & & Negative affect & $\mathrm{r}=0.33, p<0.01$ & $\begin{array}{l}\text { Intolerance of ambiguity positively correlated } \\
\text { with negative affect. }\end{array}$ \\
\hline Teoh \& Foo, (1997) & 70 & AT-20 & Entrepreneurial performance & $p<0.07$ & $\begin{array}{l}\text { TA moderates the relationship between role } \\
\text { conflict and performance measures. }\end{array}$ \\
\hline Teoh \& Foo, (1997) & 70 & AT-21 & Entrepreneurial performance & $p<0.01$ & High TA correlates with better performance. \\
\hline \multirow[t]{2}{*}{ Lal \& Hassel, (1998) } & 64 & Budner's TIA & $\begin{array}{l}\text { Perceived usefulness of } \\
\text { information characteristics of } \\
\text { management accounting } \\
\text { systems (MAS) }\end{array}$ & $p<0.05$ & $\begin{array}{l}\text { Managers with high TA perceive } \\
\text { non-conventional MAS as more useful when } \\
\text { perceived environmental uncertainty is high than } \\
\text { those with low TA. }\end{array}$ \\
\hline & & & $\begin{array}{l}\text { Perceived usefulness of } \\
\text { information in accounting } \\
\text { systems (MAS) }\end{array}$ & $p<0.01$ & $\begin{array}{l}\text { TA has a stronger effect on MAS in large firms } \\
\text { than small firms. }\end{array}$ \\
\hline \multirow[t]{2}{*}{$\begin{array}{l}\text { Firoozabadi, \& Bahredar, } \\
\qquad(2006)\end{array}$} & 240 & Budner's TIA & $\begin{array}{l}\text { Medical students } \\
\text { demographics }\end{array}$ & $p<0.05$ & Men scored lower than women on the TA scale. \\
\hline & & & Medical speciality preference & $\mathrm{n} / \mathrm{a}$ & $\begin{array}{l}\text { There was no difference in TA level between } \\
\text { medical speciality preferences. }\end{array}$ \\
\hline \multirow[t]{4}{*}{$\begin{array}{l}\text { Tapanes, Smith, \& White, } \\
\text { (2009) }\end{array}$} & 66 & $\begin{array}{l}\text { Hofstede's Value } \\
\text { Survey }\end{array}$ & $\begin{array}{l}\text { Perceived effect of dissonance } \\
\text { in online learning }\end{array}$ & $\begin{array}{l}p=0.002 \\
p=0.015\end{array}$ & $\begin{array}{l}\text { Learners from low TA cultures felt it was } \\
\text { important for their instructors to take into act } \\
\text { their cultural background and that they be } \\
\text { informed about differences between } \\
\text { their culture and that of the course. }\end{array}$ \\
\hline & & & $\begin{array}{l}\text { Perceived effect of dissonance } \\
\text { in online learning }\end{array}$ & $p=0.007$ & $\begin{array}{l}\text { High TA cultures reported being more motivated } \\
\text { to learn whilst low TA cultures were intimated. }\end{array}$ \\
\hline & & & $\begin{array}{l}\text { Perceived effect of dissonance } \\
\text { in online learning }\end{array}$ & $p=0.047$ & $\begin{array}{l}\text { High TA cultures had higher participation rates } \\
\text { (controlling for language). }\end{array}$ \\
\hline & & & $\begin{array}{l}\text { Perceived effect of dissonance } \\
\text { in online learning }\end{array}$ & $\begin{array}{l}p=0.168 ; p=0.05 \\
p=0.216 ; p=0.212\end{array}$ & $\begin{array}{l}\text { No significant differences regarding their } \\
\text { instructor's awareness, consideration culture, } \\
\text { silenced experiences and feelings of } \\
\text { alienation for the TA dimension. }\end{array}$ \\
\hline
\end{tabular}




\section{A. FURNHAM, J. MARKS}

\section{Continued}

\begin{tabular}{|c|c|c|c|c|c|}
\hline Chong, (1998) & 63 & AT-20 & $\begin{array}{l}\text { Managerial perforance } \\
\text { (via management accounting } \\
\text { systems (MAS) information) }\end{array}$ & $\begin{array}{l}\text { TA and MAS, } \\
\mathrm{r}=0.381 \text { MAS X } \\
\text { TA: } p=0.012\end{array}$ & $\begin{array}{l}\text { TA is negatively correlated with MAS, which has } \\
\text { a direct effect on managerial perfomance. }\end{array}$ \\
\hline $\begin{array}{l}\text { Hartmann \& Slapnicar, } \\
\text { (2012) }\end{array}$ & 178 & AT-20 & $\begin{array}{l}\text { Justice perceptions with the use } \\
\text { of outcome measures }\end{array}$ & $\mathrm{r}=0.318, p<0.001$ & $\begin{array}{l}\text { Managers with low TA judge an evaluation } \\
\text { process more fairly. }\end{array}$ \\
\hline \multirow[t]{2}{*}{$\begin{array}{l}\text { Swami, Stieger, Pietschnig } \\
\text { \& Voracek, (2010) }\end{array}$} & 240 & MAT-50 & Preference for surrealist art & $\begin{array}{l}\mathrm{r}=-0.22, p<0.05 \\
\mathrm{r}=-0.22, p<0.05 \\
\mathrm{r}=-0.25, p<0.001\end{array}$ & $\begin{array}{l}\text { TA positively correlates with a liking for } \\
\text { surrealist art (TA subscales: Philosophy, } \\
\text { Problem-solving, Art Forms). }\end{array}$ \\
\hline & & & Preference for surrealist films & $\begin{aligned} \mathrm{r} & =-0.19, p<0.05 \\
\mathrm{r} & =-0.18, p<0.05\end{aligned}$ & $\begin{array}{l}\text { TA positively correlates with a liking for } \\
\text { surrealist films (TA subscales: Problem-solving, } \\
\text { Art Forms). }\end{array}$ \\
\hline Rong \& Grover, (2009) & 126 & MAT-50 & $\begin{array}{l}\text { Technological knowledge } \\
\text { renewal effectiveness }\end{array}$ & $(\mathrm{t}=2.32, p=0.01)$ & $\begin{array}{l}\text { TA has a positive impact on technological } \\
\text { knowledge renewal effectiveness. }\end{array}$ \\
\hline $\begin{array}{l}\text { Iyer, McBride, \& Reckers, } \\
\text { (2012) }\end{array}$ & 78 & AT-20 & $\begin{array}{l}\text { Capital investment proposal } \\
\text { recommendation } \\
\text { (with/without a decision aid) }\end{array}$ & $\mathrm{F}=5.09, p=0.027$ & $\begin{array}{l}\text { Low TA Ss applied decision aids when making a } \\
\text { decision on an ambiguous investment. }\end{array}$ \\
\hline \multirow[t]{8}{*}{ Buhr \& Dugas, (2006) } & 197 & Budner's TIA & Intolerance of uncertainty & $\mathrm{r}=0.42, p<0.001$ & $\begin{array}{l}\text { TA has a positive correlation with tolerance of } \\
\text { uncertainty. }\end{array}$ \\
\hline & & & Worry & $\mathrm{r}=0.27, p<0.001$ & TA has a negative relationship with worry. \\
\hline & & & Self-oriented perfectionism & $\mathrm{r}=0.19, p<0.01$ & $\begin{array}{l}\text { TA has a negative relationship with self-oriented } \\
\text { perfectionism. }\end{array}$ \\
\hline & & & $\begin{array}{l}\text { Socially-prescribed } \\
\text { perfectionism }\end{array}$ & $\mathrm{r}=0.35, p<0.001$ & $\begin{array}{l}\text { TA has a negative relationship with } \\
\text { socially-prescribed perfectionism. }\end{array}$ \\
\hline & & & Other-oriented perfectionism & $\mathrm{r}=0.15, p<0.05$ & $\begin{array}{l}\text { TA has a negative relationship with } \\
\text { other-oriented perfectionism. }\end{array}$ \\
\hline & & & Perceived mastery & $\mathrm{r}=-0.14, p<0.05$ & $\begin{array}{l}\text { TA has a positive relationship with perceived } \\
\text { mastery. }\end{array}$ \\
\hline & & & Perceived constraints & $\mathrm{r}=0.32, p<0.001$ & $\begin{array}{l}\text { TA has a negative relationship with perceived } \\
\text { constraint. }\end{array}$ \\
\hline & & & Age & $\mathrm{r}=-0.24, p<0.01$ & TA increases with age. \\
\hline \multirow[t]{3}{*}{$\begin{array}{l}\text { Wolfradt, Oubaid, Straube, } \\
\text { Bischoff \& Mischo, (1999) }\end{array}$} & 374 & $\begin{array}{l}\text { The Scale of } \\
\text { Interpersonal } \\
\text { Intolerance of } \\
\text { Ambiguity (SIA) by } \\
\text { Wolfradt and } \\
\text { Rademacher }\end{array}$ & $\begin{array}{l}\text { Schizotypal personality } \\
\text { disorder }\end{array}$ & $\begin{array}{l}\mathrm{r}=0.27, p<0.001 \\
\mathrm{r}=0.25, p<0.001 \\
\mathrm{r}=0.43, p<0.001\end{array}$ & $\begin{array}{l}\text { TA has a negative relationship with Schizotypal } \\
\text { personality disorder (cognitive-perceptual, } \\
\text { interpersonal and disorganizational deficits). }\end{array}$ \\
\hline & & & $\begin{array}{l}\text { Information processing } \\
\text { (need for cognition) }\end{array}$ & $\mathrm{r}=-0.19, p<0.001$ & $\begin{array}{l}\text { TA has a positive relationship with this type of } \\
\text { information processing (need for cognition). }\end{array}$ \\
\hline & & & General Self-Efficacy & $\mathrm{r}=-0.31, p<0.001$ & $\begin{array}{l}\text { TA has a positive relationship with general } \\
\text { self-efficacy. }\end{array}$ \\
\hline Furnham \& Avison, (1997) & 62 & AT-20 & Painting preferences & $\mathrm{r}=0.31, p<0.05$ & $\begin{array}{l}\text { TA is significantly related positively to surrealist } \\
\text { (fewer elements) paintings. }\end{array}$ \\
\hline $\begin{array}{l}\text { Ironside, Jefferies \& } \\
\quad \text { Martin, (2009) }\end{array}$ & $\mathrm{n} / \mathrm{a}$ & $\begin{array}{c}\text { The Multiple } \\
\text { Stimulus Types } \\
\text { Ambiguity Tolerance } \\
\text { Scale-I (MSTAT-I) }\end{array}$ & $\begin{array}{l}\text { Achievement of patient safety } \\
\text { competencies }\end{array}$ & $p>0.05$ & $\begin{array}{l}\text { TA did not correlate with nurses' patients safety } \\
\text { competencies. }\end{array}$ \\
\hline Leong \& Ward, (2000) & 106 & MSTAT-I & Identity conflict & $\mathrm{r}=-0.32, p<0.005$ & $\begin{array}{l}\text { High TA is a significant predictor of identity in } \\
\text { Chinese sojourners in Singapore. }\end{array}$ \\
\hline \multirow[t]{3}{*}{$\begin{array}{l}\text { Le, Haller, Langer, } \\
\text { Courvoisier, (2012) }\end{array}$} & 75 & Budner's TIA & Mindfulness & $\mathrm{r}=-0.35, p<0.01$ & TA positively correlated with mindfulness. \\
\hline & & & Thinking style & $\begin{array}{l}\mathrm{r}=-0.54, p<0.01 \\
\mathrm{r}=-0.43, p<0.01\end{array}$ & $\begin{array}{l}\text { TA positively correlated with thinking style } \\
\text { (mean; concrete). }\end{array}$ \\
\hline & & & Affect & $\mathrm{r}=-0.01 ; \mathrm{r}=0.13$ & $\begin{array}{l}\text { TA did not correlate with pre-experimental } \\
\text { (positive or negative) affect. }\end{array}$ \\
\hline \multirow[t]{2}{*}{$\begin{array}{l}\text { Westerberg, Singh \& } \\
\text { Häckner, (1997) }\end{array}$} & 139 & $\begin{array}{l}\text { Modified from Lorsch } \\
\text { and Morse }\end{array}$ & Firms' financial performance & $\mathrm{b}=0.26, p<0.01$ & $\begin{array}{l}\text { CEOs with high TA were related to firms with } \\
\text { high financial performance. }\end{array}$ \\
\hline & & & Firms' market performance & $\mathrm{b}=0.26, p<0.01$ & $\begin{array}{l}\text { CEOs with high TA were related to firms with } \\
\text { high market performance. }\end{array}$ \\
\hline
\end{tabular}




\section{A. FURNHAM, J. MARKS}

\section{Continued}

\begin{tabular}{|c|c|c|c|c|c|}
\hline & & & Firms' market orientation & $p>0.05$ & $\begin{array}{l}\text { CEOs' TA did not correlate with firms that } \\
\text { emphasize planning orientation. }\end{array}$ \\
\hline & & & Firms' planning orientation & $p>0.05$ & $\begin{array}{l}\text { CEOs' TA did not correlate with firms that } \\
\text { emphasize market orientation. }\end{array}$ \\
\hline \multirow[t]{3}{*}{$\begin{array}{l}\text { Richardson, Jain \& Dick, } \\
\text { (1996) }\end{array}$} & 582 & Budner's TIA & Private brand proneness & $p>0.05$ & $\begin{array}{l}\text { TA did not correlate with private brand } \\
\text { proneness. }\end{array}$ \\
\hline & & & $\begin{array}{l}\text { Value for money perceptions of } \\
\text { private label brands. }\end{array}$ & $\beta=-0.117, p<0.05$ & $\begin{array}{l}\text { TA positively correlates with money perceptions } \\
\text { (value for money). }\end{array}$ \\
\hline & & & $\begin{array}{l}\text { Reliance on extrinsic cues in } \\
\text { quality assessment. }\end{array}$ & $\beta=0.251, p<0.05$ & $\begin{array}{l}\text { TA had a negative relationship with reliance on } \\
\text { extrinsic cues in quality assessment. }\end{array}$ \\
\hline \multirow[t]{2}{*}{$\begin{array}{c}\text { Altinay, Madanoglu, } \\
\text { Daniele \& Lashley, (2012) }\end{array}$} & 205 & $\begin{array}{l}\text { Acedo and Jones } \\
\text { scale: modified from } \\
\text { Lorsch and Morse }\end{array}$ & Intention to start a business & $\mathrm{r}=0.274, p>0.05$ & $\begin{array}{l}\text { There was no relationship between TA and } \\
\text { intention to start a business. }\end{array}$ \\
\hline & & & Risk-taking propensity & $\mathrm{r}=0.318, p=0.426$ & $\begin{array}{l}\text { There was a positive relationship between } \\
\text { tolerance of ambiguity and risk taking propensity. }\end{array}$ \\
\hline \multirow[t]{8}{*}{ Caligiuri, Tarique, (2012) } & 641 & $\begin{array}{l}\text { Modified Gupta and } \\
\text { Govindarajan (1984) }\end{array}$ & $\begin{array}{l}\text { Non-work cross-cultural } \\
\text { experiences }\end{array}$ & $\mathrm{r}=0.28, p<0.01$ & $\begin{array}{l}\text { TA correlated positively with non-work } \\
\text { cross-cultural experiences. }\end{array}$ \\
\hline & & & $\begin{array}{l}\text { Organization-initiated } \\
\text { cross-cultural experiences }\end{array}$ & $\mathrm{r}=0.06, p<0.01$ & $\begin{array}{l}\text { TA correlated positively with "organization- } \\
\text { initiated" cross cultural experiences. }\end{array}$ \\
\hline & & & $\begin{array}{l}\text { Dynamic cross-cultural } \\
\text { competencies }\end{array}$ & $\mathrm{R}^{2}=0.26, p<0.01$ & $\begin{array}{l}\text { TA correlated positively with dynamic } \\
\text { cross-cultural competencies. }\end{array}$ \\
\hline & & & Neuroticism & $\mathrm{r}=0.07, p>0.05$ & TA did not correlate with neuroticism. \\
\hline & & & Extraversion & $\mathrm{r}=0.37, p<0.01$ & $\begin{array}{l}\text { TA had a strong, positive correlation with } \\
\text { extraversion. }\end{array}$ \\
\hline & & & Agreeableness & $\mathrm{r}=-0.19, p<0.05$ & TA correlated negatively with agreeableness. \\
\hline & & & Openness & $\mathrm{r}=0.29, p<0.01$ & $\begin{array}{l}\text { TA had a strong, positive correlation with } \\
\text { openness. }\end{array}$ \\
\hline & & & Conscientiousness & $\mathrm{r}=0.00, p>0.05$ & TA did not correlate with conscientiousness. \\
\hline \multirow[t]{2}{*}{ Neill \&Rose, (2007) } & 167 & Modified MSTAT-I & Equivocality & $\mathrm{t}=0.27, p<0.01$ & $\begin{array}{l}\text { An organisations TA correlates with } \\
\text { equivocality. }\end{array}$ \\
\hline & & & $\begin{array}{l}\text { Market-focused } \\
\text { strategic flexibility }\end{array}$ & $\mathrm{t}=0.21, p<0.01$ & $\begin{array}{l}\text { An organizations TA is positively correlated with } \\
\text { market-focused strategic flexibility. }\end{array}$ \\
\hline \multirow[t]{7}{*}{$\begin{array}{l}\text { Rajagopal \& Hamouz, } \\
\qquad(2009)\end{array}$} & 111 & Budner's TIA & $\begin{array}{l}\text { Willingness to try (A factor of } \\
\text { the Food Attitude Behavior } \\
\text { Openness Scale (FABOS)) }\end{array}$ & $\mathrm{r}=0.332, p<0.01$ & $\begin{array}{l}\text { TA positively correlated with the factor } \\
\text { "willingness to try". }\end{array}$ \\
\hline & & & Seeking novelty (FABOS) & $\mathrm{r}=0.447, p<0.01$ & $\begin{array}{l}\text { TA positively correlated with the factor } \\
\text { "seeking novelty". }\end{array}$ \\
\hline & & & Enjoy novelty (FABOS) & $\mathrm{r}=0.212, p>0.01$ & $\begin{array}{l}\text { TA did not correlate with the "enjoy novelty" } \\
\text { factor. }\end{array}$ \\
\hline & & & Conditional openess (FABOS) & $\mathrm{r}=0.098, p>0.01$ & $\begin{array}{l}\text { TA did not correlate with the "conditional } \\
\text { openness" factor. }\end{array}$ \\
\hline & & & Internationalization decisions & $p<0.05$ & $\begin{array}{l}\text { CEOs' international orientation did not relate to } \\
\text { TA. }\end{array}$ \\
\hline & & & $\begin{array}{l}\text { Risk associated with } \\
\text { internationalization }\end{array}$ & $p<0.001$ & $\begin{array}{l}\text { CEOs with high TA perceived lower levels of } \\
\text { risk. }\end{array}$ \\
\hline & & & $\begin{array}{l}\text { Proactive disposition to } \\
\text { internationalization }\end{array}$ & $p<0.05$ & $\begin{array}{l}\text { TA did not correlate with a proactive disposition } \\
\text { to internationalization. }\end{array}$ \\
\hline Carter \& Hall, (2008) & 279 & Budner's TIA & $\begin{array}{l}\text { Observational Test of } \\
\text { Ecological Sensitivity }\end{array}$ & $p<0.05$ & $\begin{array}{l}\text { TA and openness to experience gave a combined } \\
\text { score to assess cognitive openess, which } \\
\text { correlated significantly positively with the } \\
\text { Observational Test of Ecological Sensitivity. }\end{array}$ \\
\hline $\begin{array}{l}\text { Gurel, Altinay, \& Daniele, } \\
\text { (2010) }\end{array}$ & 206 & $\begin{array}{l}\text { Acedo and Jones } \\
(2007)=\text { modified } \\
\text { Lorsch and Morse } \\
\quad(1974)\end{array}$ & Entrepreneurial intention & $p>0.05$ & $\begin{array}{l}\text { TA was not associated with intentions to start a } \\
\text { business. }\end{array}$ \\
\hline
\end{tabular}


Table 2.

Table showing measures of the TA scales.

\begin{tabular}{|c|c|c|c|c|}
\hline Author & Name of scale & $\mathrm{N}$ & No. of items & Dimensions \\
\hline $\begin{array}{l}\text { Herman, Stevens, Bird, Mendenhall, Oddou, } \\
\text { (2010) }\end{array}$ & The tolerance of ambiguity scale & 2351 & 12 & (1) 4 \\
\hline McLain (2009) & $\begin{array}{l}\text { Multiple stimulus types ambiguity tolerance scale-II } \\
\text { (MSTAT-II) }\end{array}$ & 870 & 13 & (1) 3 \\
\hline Buhr \& Dugas (2002) & Intolerance of ambiguity scale & 276 & 27 & 4 \\
\hline Lange \& Houran (1999) & Rasch model AT-20 & 110 & 18 & 1 \\
\hline Durrheim \& Foster (1996) & Attitudinal ambiguity tolerance scale & 421 & 45 & 4 \\
\hline McLain (1993) & Multiple stimulus types ambiguity tolerance scale-I & 148 & 22 & 1 \\
\hline Norton (1975) & MAT 50 & 1496 & 61 & 8 \\
\hline MacDonald (1970) & AT-20 & 789 & 20 & 1 \\
\hline Budner (1962) & 16 item scale & 947 & 16 & 1 \\
\hline O’Connor (1952) & Walk & Unpublished & 8 & 1 \\
\hline
\end{tabular}

0.71 and with 105 students over 2 months $r=0.57$ but there was no evidence of the test's internal reliability. The test was in part validated with the use of semantic differential ratings of contradictory and non-contradictory adjective-noun concept combinations (Rydell, 1966). MacDonald (1970) however, attempted some psychometric evaluation of the Rydell-Rosen scale but added 4 extra items. This larger scale had a test-retest reliability of 0.63 over 6 months and was cross-validated on nearly 800 undergraduates. The test was correlated with Rokeach's dogmatism scale, the Gough-Sanford Rigidity scale and church attendence but not social desirability. The split-half reliability was also satisfactory at 0.73 , and MacDonald noted that it"shows promise of being a useful instrument for the measurement and further investigation of ambiguity tolerance" (p. 797).

Lange and Houran (1999) praised the AT-20 scale for its convergent validity and internal consistency, but argued that an Item Response Theory (ITR) framework have more appropriate scaling properties for use in structural modeling.

In particular, they propose the Rasch (1960) model because "Rasch scaling requires no iterative estimation procedures" (p.468). The Rasch model AT-20 (Lange \& Houran, 1999) only differentiates itself from the AT-20 from a scaling point of view-there are no additional items but two were removed. It provides no new information for evidence of validity. Results showed that the positive item-rest point biserial correlations provide evidence of the scale's uni-dimensionality, correlations were consistent with the local independence assumption, the discrimination parameter values show the data fits with the Rasch model and the person fit to the Rasch model was satisfactory. The sample size was large enough to yield an adequate item separation value (3.72), which indicates the estimated item locations have a KR-20 reliability index of 0.93 . The internal consistency was 0.68 (KR-20), which is slightly lower than the value reported by MacDonald (1970) but still just about satisfactory considering the number of items. The Rasch approach is said to differ to the classical test theory with respect to estimating tolerance of ambiguity and assessing the error of measurement associated with such estimates.

In the mid-1970s Norton (1975) argued that the extant pa- per-and-pencil measures of TA were "flawed by low internal reliability and the absence of adequate validity evidence" (p. 607). This he believed was in part due to ambiguities associated with the term ambiguous which was used in 8 different ways to describe: multiple meanings; vagueness, incompleteness, fragmentation; as a probability; unstructured; lack of information; uncertainty; inconsistencies, contradiction, contraries and unclearness. He therefore developed a 50-item "measure" (MAT50) which was tested seven times to develop high reliability which was $r=0.38$ (Kuder-Richardson) and with a test-retest reliability of 0.86 after $10-12$ weeks. He also attempted to determine 3 types of validity: content validity (through content analysis and faking studies), criterion-related (through correlations with measures of dogmatism and rigidity) and construct validity (through measures of willingness to volunteer for an ambiguous study; aesthetic judgement; a content analysis of verbal behaviour and behavioural dramatisation). As predicted, high TA Ss tended to volunteer more for undefined experiments, to use different aesthetic judgements and be more dramatic in problem-solving groups.

Norton (1975) ended his paper suggesting seven research questions the scale may be used to investigate most of which had been tested before but not when using a self-report TA measure: what is the cognitive process during an ambiguous situation; how can the information theorist account for ambiguity; is it possible to use the measure of TA to help identify therapeutic problems; do groups prefer leaders who are TA; how is trusting behaviour related to TA: to what degree is TA culture bound; what is the function of TA in a conflict resolution situation.

Nearly 40 years ago Lorsch and Morse (1974) argued that managers who often face ambiguous decisions have to be more willing to take risks. They developed a 7-item scale with the aim to test members of organizations, specifically managers' TA. Therefore this scale has been prominent mainly in business journals and has been modified a number of times in order to fit more appropriately with researchers' needs (Westerberg, Singh \& Häckner, 1997; Acedo \& Jones, 2007; Gurel, Altinay \& Daniele, 2010; Caligiuri \& Tarique, 2012). Among others, Gupta and Govindarajan (1984) reduced the number of items to 
4, and reported an internal reliability of 0.57 .

Motivated by the psychometric weakness of widely used measures of TA, McLain (1993) developed a new 22-item measure called the Multiple Stimulus Types Ambiguity Tolerance (MSTAT). McLain attempted to redefine TA so that the three conceptual perspectives of TA could be separately defined and integrated. These three concepts are: TA as a source of threat from novel, complex and insoluble stimuli (Budner, 1962), ambiguity as term for second order probability (Ellsberg, 1961), and TA as a link to the authoritarianism family (Frenkel-Brunswik, 1949). McLain defined TA as "a range, from rejection to attraction, of reactions to stimuli perceived as unfamiliar, complex, dynamically uncertain, or subject to multiple conflicting interpretations" (p. 184). A factor analysis of 148 respondents supported a uni-dimensional model, a general tolerance for ambiguity. The scale was found to have good internal consistency, $\alpha=0.86$. Evidence of the scale's concurrent validity for the scale comes from significant positive correlations with other TA scales (Budner's (1962) 16-item scale, Storey and Aldag's (1983) 8-item scale and MacDonald's (1970) 20-item scale as well as significantly correlating with willingness to take risks, receptivity to change and a negative correlation with dogmatism. The adequate psychometric properties and refined construct of this scale make it one of the more popular measures in recent times.

In 2009, McLain refined the MSTAT scale. The MSTAT-II is a 13-item scale derived from the original 22 items. The reduced number of items means that respondents use less cognitive resources completing the questionnaire. Items were removed from the MSTAT-I on the basis of feedback from researchers and respondents who used the questionnaire. The remaining items were kept if they added to the overall construct, correlated with the scale, and did not confound it through context-specificity or incomprehensibility. The data was collected from a sample of university students and firefighter-emergency medical technicians $(\mathrm{n}=870)$. The internal consistency reliability was 0.83 , which is good despite being slightly lower than the MSTAT-I. Three factors were identified by a factor analysis, however a scree plot showed a distinct first factor only. This factor corresponded to ambiguity tolerance in general and confirmatory factor analysis suggested the one-dimensional theoretical model is appropriate.

McLain found that MSTAT-II correlated significantly and positively with MacDonald's AT-20, sensation seeking, perceived risk, perceived uncertainty, which provides evidence for concurrent validity. The scale correlated negatively with somatic tension and social desirability. The correlation with Budner's scale however, was not significant. McLain argued that this finding may be due to the Budner scale's low reliability and poor item wording. He later found that the Budner scores had a multidimensional structure and therefore should not be seen to undermine the strength of the MSTAT-II. Despite these arguments, it should be noted that the MSTAT-I did significantly correlate with the Budner scale and it may be possible that because items have been removed, the MSTAT-II is less comprehensive. Therefore this scale is recommended over the MSTAT-I when space is limited or when participants could potentially become cognitively overloaded.

Durrheim and Foster (1997) did not conceive TA as psychological trait, but as a content specific construct. This is consistent with Frenkel-Brunswick's (1949) original construct of TA as an "attitudinal variable", which was not assumed to general- ise across different social objects. Among others, Durrheim and Foster (1997) proposed that intercorrelations of TA measures are "spurious relationships between shared attitudinal scale content" (p. 741) and that the relationship is a methodological artefact. This accounts for the failure to correlate different experimental procedures (e.g. Million, 1957). They argue that factor analysis (e.g. Furnham, 1994) show TA to be multidimensional and conclude that there is little evidence for regarding TA as a stable and generalised personality trait.

Durrheim and Foster (1997), who are social, not personality, psychologists, developed the Attitudinal Ambiguity Tolerance scale (AAT) in response to these objections, which is based on the uni-polar scaling procedure that was originated by Kaplan (1972) and subsequently used by Scott and colleagues to assess ambivalence (Scott, 1966, 1969; Scott, Osgood, \& Peterson, 1979). The scale uses a wide range of authority figures as scale items and participants are asked to express their degree of ambivalence. This scale measures evaluative performance and is therefore thought to model Frenkel-Brunswick's original description of prejudiced and non-prejudiced children. The scale was found to have an adequate internal reliability $(\alpha=0.81)$ and test-retest reliability $(\mathrm{r}=0.66)$. A factor analysis revealed four factors, which suggests a multi-dimensional structure and supports the original hypothesis. Evidence for concurrent validity for this scale comes from a significant positive correlation with the Ambivalence scale that the AAT was based on.

The AAT scale was not significantly correlated with Budner's scale, however this is inconclusive because not only is the Budner scale thought to lack reliability and validity, but there are theoretical underlying differences between the two scales. Criterion groups were also used to validate the AAT scale because of the political context in South Africa at the time of testing. Although Durrheim and Foster's criticisms of the TA concept seem valid, they have largely been ignored by contemporary research for three reasons. Firstly, the focus of the TA concept has shifted away from prejudice and authoritarianism and moved towards reactions in response to insufficient information. Secondly, the psychometric qualities of TA scales have increased. Third, empirical research supports a one-dimensional theoretical model (McLain, 2009; Benjamin et al. 1996; Furnham \& Ribchester, 1995), which describes TA as "unitary yet multifaceted" (Herman et al., 2010: p. 59).

It should be noted that Wolfradt and Rademacher (1999) developed and validated a scale for interpersonal TA. This scale was designed for use as a clinical tool. The scale is not widely used however, despite good internal reliability (Cronbach's alpha $=0.86$; Wolfradt, Oubaid, Straube, Bischoff, \& Mischo, 1999).

Herman et al. (2010) proposed a new measure of TA that aimed to better understand its link to cross-cultural phenomena, improved conceptual dimensionality and psychometric evidence. They attributed the disagreement in construct dimensionality to the diversity of research contexts, arguing that overly general items may not be suitable for all the diverse concepts of TA. The MSTAT-II may suffer from over-generalisation, although the author admitted this himself (McLain, 2009).

Instead, Herman et al. (2010) reasoned that context-dependent measures should be developed in areas that may have problems if they use the generalised conception. Their measure focuses on cross-cultural contexts. They used Budner's (1962) conceptualisation and measure of TA (because it has been so 
influential despite its flaws) as a basis on which they performed an exploratory factor analysis, assessment of internal consistency and item-total correlations, then added and removed items to improve the measure $(\mathrm{n}=2351)$. The new items were designed to fit with prior conceptions of TA (Budner, 1962; McLain, 1993) and relevant to a cross-cultural context. They found the overall internal consistency to be acceptable $(\alpha=$ 0.73 ). Factor analysis distinguished 4 factors, but the measure was found to fit a one-dimensional theoretical framework because the internal consistencies of the individual dimensions were not high enough to support multidimensionality. The four factors were; valuing diverse others, change, challenging perspectives and unfamiliarity. Valuing diverse others has not appeared in other recent conceptualisation (McLain, 1993, 2009; Furnham, 1994), which the authors relate to the interpersonal nature of cross-cultural settings. This 12-item scale is a useful tool for measuring TA in cross-cultural contexts and it may revolutionise the measurement of TA, starting a trend in the development of context-specific measures.

It should be noted that Wolfradt and Rademacher (1999) developed and validated a scale for interpersonal TA. This scale was designed for use as a clinical tool. The scale is not widely used however, despite good internal reliability (Cronbach's alpha $=0.86$; Wolfradt, Oubaid, Straube, Bischoff, \& Mischo, 1999).

\section{Conclusion}

The TA concept has gone through changes since its conception in 1948, when it was synonymous with authoritarianism and prejudice. The focus has now shifted to reflect the contemporary definition of ambiguity (Ellsberg, 1962). Researchers have ducked the questions about where TA sits in Big Five Factor space. Is TA a second or third order facet or does it belong outside the big five a little like Locus of Control or other "cognitive personality variables"?

The papers in this area still lack sophistication. For instance there appear to be no studies that have attempted to determine the heritability of TA. This would help differentiate between the social psychological conception of TA as a set of attitudes vs the differential psychology conception of a stable, perhaps even biologically based trait. Nor have the tests been frequently subjected to structural equation modelling to determine both the facets or factors of TA as well as its determinants. However, the interest among clinicians in the TU concept may suggest that it is closely linked positively to Neuroticism and negatively to Openness-to-Experience.

The interest in TA seems to have shifted from differential and social psychologist to clinical and organisational psychologists who see it as measure of adaptation and healthy functioning. There yet remains a need to do some psychometric housekeeping as done by Furnham (1994) to look at the relationship between the existent measures, but perhaps more importantly to integrate TA measures and theory to modern psychometric methods as well as cognitive neuro-science.

\section{REFERENCES}

Acedo, F. J., \& Jones, M. V. (2007). Speed of internationalisation and entrepreneurial cognition. Journal of World Business, 42, 236-252. doi:10.1016/j.jwb.2007.04.012

Altinay, L., Madanoglu, M., Daniele, R., \& Lashley, C. (2012). The influence of family tradition and psychological traits on entrepreneu- rial intention. International Journal of Hospitality Management, 31, 489-499. doi:10.1016/j.ijhm.2011.07.007

Anderson, S., \& Schwartz, A. (1992). Intolerance of ambiguity and depression. Social Cognition, 10, 271-298.

doi:10.1521/soco.1992.10.3.271

Bardi, A., Guerra, V. M., Sharadeh, G., \& Ramdeny, D. (2009). Openness and ambiguity intolerance: Their differential relations to wellbeing in the context of an academic life transition. Personality and Individual Differences, 47, 219-223. doi:10.1016/j.paid.2009.03.003

Becherer, R. C., \& Maurer, J. G. (1999). The proactive personality disposition and entrepreneurial behavior among small company presidents. Journal of Small Business Management, 37, 28-36.

Benjamin, A. J., Riggio, R. E., \& Mayes, B. T. (1996). Reliability and factor structure of Budner's Tolerance for Ambiguity Scale. Journal of Social Behavior and Personality, 11, 625-632.

Birrell, J., Meares, K., Wilkinson, A., \& Freeston, M. (2011). Toward a definition of intolerance of uncertainty. Clinical Psychology Review, 31, 1198-1208. doi:10.1016/j.cpr.2011.07.009

Budner, S. (1962). Intolerance of ambiguity as a personality variable. Journal of Personality, 30, 29-50. doi:10.1111/j.1467-6494.1962.tb02303.x

Buhr, K., \& Dugas, M. J. (2006). Investigating the construct validity of intolerance of uncertainty and its unique relationship with worry. Journal of Anxiety Disorders, 20, 222-236. doi:10.1016/j.janxdis.2004.12.004

Caligiuri, P. M., Jacobs, R. R., \& Farr, J. L. (2000). The attitudinal and behavioural openness scale: Scale development and construct validation. International Journal of Intercultural Relations, 24, 27-46. doi:10.1016/S0147-1767(99)00021-8

Caligiuri, P., \& Tarique, I. (2012). Dynamic cross-cultural competenvies and gobal leadership effectiveness. Journal of World Business, in press. doi:10.1016/j.jwb.2012.01.014

Carleton, R. N., Norton, M. A. P., \& Asmundson, G. J. G. (2007). Fearing the unknown: A short version of the intolerance of uncertainty scale. Journal of Anxiety Disorders, 21, 105-117. doi:10.1016/j.janxdis.2006.03.014

Carter, J. D., \& Hall, J. A. (2008). Individual differences in the accuracy of detecting social covariations: Ecological sensitivity. Journal of Research in Personality, 42, 439-455. doi:10.1016/j.jrp.2007.07.007

Chong, V. K. (1998). Testing the contingency (fit) between management accounting systems and the managerial performance: A research note on the moderating role of tolerance of ambiguity. British Accounting Review, 30, 331-342. doi:10.1006/bare.1998.0073

Conchar, M. P., Zinkhan, G. M., Peters, C., \& Olavarrieta, S. (2004). An integrated framework for the conceptualization of consumers' perceived risk processing. Journal of the Academy of Marketing Science, 32, 418-436. doi:10.1177/0092070304267551

DeRoma, V. M., Martin, K. M., \& Kessler, M. L. (2003). The relationship between tolerance for ambiguity and need for course structure. Journal of the Institute of Psychology, 30, 104-109.

Diaz, F., \& Rodriguez, A. (2003). Locus of control and values of community entrepreneurs. Social Behavior and Personality, 31, 739-748. doi:10.2224/sbp.2003.31.8.739

Dugas, M. J., Freeston, M. H., \& Ladouceur, R. (1997). Intolerance of uncertainty and problem orientation in worry. Cognitive Therapy and Research, 21, 593-606. doi:10.1023/A:1021890322153

Durrheim, K., \& Foster, D. (1997). Tolerance of ambiguity as a content specific construct. Personality and Individual Differences, 22, 741750. doi:10.1016/S0191-8869(96)00207-3

Ehrman, M. E. (1999). Ego boundaries and tolerance of ambiguity in second language learning. In J. Arnold (Ed.), Affect in Language Learning (pp. 68-86). Cambridge, New York.

Ellsberg, D. (1961). Risk, ambiguity, and the Savage axioms. Quarterly Journal of Economics, 75, 643-669. doi:10.2307/1884324

Endres, M. L., Chowdhury, S., \& Milner, M. (2009) .Ambiguity tolerance and accurate assessement of self-efficacy in a complex decision task. Journal of Management and Organization, 15, 31-46. doi:10.5172/jmo.837.15.1.31

Entrialgo, M., Fernandez, E., \& Vazquez, C. J. (2000). Psychological characteristics and process: The role of entrepreurship in Spanish 
SMEs. European Journal of Innovation Management, 3, 137-149. doi:10.1108/14601060010334894

Eysenck, H. (1954). The psychology of politics. London: Routledge.

Facione, N. C., Facione, P. A., \& Sanchez, C. A. (1994). Critical thinking disposition as a measure of competent clinical judgment: The development of the California critical thinking disposition inventory. Journal of Nursing Education, 33, 345-350.

Feather, N. (1969). Preference for information in relation to consistency, novelty, intolerance of ambiguity and dogmatism. Australian Journal of Psychology, 31, 235-249. doi:10.1080/00049536908257793

Fergus, T., \& Wu, K. (2013). The intolerance of uncertainty scale. Assessment.

Firoozabadi, A., \& Bahredar, M. J. (2006). P02-419-Ambiguity tolerance in students entering medical school (SHIRAZ-2006). European Psychiatry, 26, 1015. doi:10.1016/S0924-9338(11)72720-1

Freeston, M. H., Rhéaume, J., Letarte, H., Dugas, M. J., \& Ladouceur, R. (1994). Why do people worry? Personality and Individual Differences, 17, 791-802. doi:10.1016/0191-8869(94)90048-5

Frenkel-Brunswick, E. (1948). Intolerance of ambiguity as an emotional and perceptual personality variable. Journal of Personality, 18, 108-123. doi:10.1111/j.1467-6494.1949.tb01236.x

Frenkel-Brunswick, E. (1949). Tolerance toward ambiguity as a personality variable. American Psychologist, 3, 268.

Frenkel-Brunswick, E. (1951). Personality theory and perception. In R. Blake, \& E. Ramsey (Eds.), Perception: An approach to personality. New York: Ronald. doi:10.1037/11505-013

Furnham, A. (1994). A content, correlational and factor-analytic study of 4 tolerance of ambiguity questionnaires. Personality and Individual Differences, 16, 403-410. doi:10.1016/0191-8869(94)90066-3

Furnham, A., \& Avison, M. (1997). Personality and preference for surreal paintings. Personality and Individual Differences, 23, 923-935. doi:10.1016/S0191-8869(97)00131-1

Furnham, A., \& Ribchester, T. (1995). Tolerance of ambiguity: A review of the concept, its measurement and applications. Current Psychology, 14, 179-199. doi:10.1007/BF02686907

Geller, G., Tambor, E. S., Chase, G. A., \& Holtzman, N. A. (1993). Measuring physicians' tolerance for ambiguity and its relationship to their reported practices regarding genetic testing. Medical Care, 31, 989-1001. doi:10.1097/00005650-199311000-00002

Goldratt, E. (2000). The impact of perceived uncertainty, tolerance for ambiguity and feelings of stress on employees attitudes towards organizational change. Unpublished doctoral dissertation, Bar-Ilan University, Ramat Gan.

Green V., \& Roger, D. (2001). Coping with uncertainty: The construction of a new measure. Personality and Individual Differences, 31 , 519-534. doi:10.1016/S0191-8869(00)00156-2

Grenier, S., Barrette, A. M., \& Ladouceur, R. (2005). Intolerance of uncertainty and intolerance of ambiguity: Similarities and differences. Personality and Individual Differences, 39, 593-600. doi:10.1016/j.paid.2005.02.014

Gupta, A., \& Govindarajan, V. (1984). Business unit strategy, managerial characteristics and business unit effectiveness at strategy implementation. Academy of Management Journal, 27, 25-41. doi:10.2307/255955

Gurel, E., Altinay, L., \& Daniele, R. (2010). Tourism students' entrepreneurial intentions. Annals of Tourism Research, 37, 646-669. doi:10.1016/j.annals.2009.12.003

Hartmann, F., \& Slapnicar, S. (2012). The perceived fairness of performance evaluation: The role of uncertainty. Management Accounting Research, 23, 17-33. doi:10.1016/j.mar.2011.10.004

Hartmann, F. G. H. (2005). The effects of tolerance for ambiguity and uncertainty on the appropriateness of accounting performance measures. ABACUS, 41, 241-264. doi:10.1111/j.1467-6281.2005.00181.x

Hazen, B. T., Overstreet, R. E., Jones-Farmer, L. A., \& Field, H. S. (2012). The role of ambiguity tolerance in consumer perception of remanufactured products. International Journal of Production Economics, 135, 781-790. doi:10.1016/j.ijpe.2011.10.011

Herman, J. L., Stevens, M. J., Bird, A., Mendenhall, M., \& Oddou, G. (2010). The tolerance for ambiguity scale: Towards a more refined measure for international management research. International Journal of Intercultural Relations, 34, 58-65. doi:10.1016/j.ijintrel.2009.09.004

Hofstede, G. (1984). Cultures' consequences. Beverly Hills, CA: Sage. Hofstede, G., \& Bond, M. H. (1984). Hofstede's cultural dimensions: An independent validation using Rockeach's value survey. Journal of Cross-Cultural Psychology, 15, 417-433. doi: $10.1177 / 0022002184015004003$

Houran, J., \& Williams, C. (1998). Relation of tolerance of ambiguity to global and specific paranormal experience. Psychological Reports, $83,807-818$.

Hung, K.-T., \& Tangpong, C. (2010). General risk propensity in multifaceted business decisions: Scale development. Journal of Managerial Issues, 22, 88-106.

Ironside, P. M., Jefferies, P. R., \& Martin, A. (2009). Fostering patient safety competencies using multiple-patient simulation experiences. Nursing Outlook, 57, 332-337. doi:10.1016/j.outlook.2009.07.010

Iyer, G., McBride, D., \& Reckers, P. (2012). The effect of a decision aid on risk aversion in capital investment decisions. Advances in Accounting, 28, 64-74. doi:10.1016/j.adiac.2012.02.007

Jaensch, E. (1938). Der gegenryppus. Leipzig: Barth.

Judge, T. A., Thoresen, C. J., Pucik, V., \& Welbourne, T. M. (1999). Managerial coping with organizational change: A dispositional perspective. Journal of Applied Psychology, 84, 107-122. doi:10.1037/0021-9010.84.1.107

Kaplan, K. J. (1972). On the ambivalence-indifference problem in attitude theory and measurement: A suggested modification of the semantic differential technique. Psychological Bulletin, 77, 361-372. doi: $10.1037 / \mathrm{h} 0032590$

Krohne, H. W. (1989). The concept of coping modes: Relating cognitive person variables to actual coping behaviour. Advances in Behaviour Research and Therapy, 11, 235-248.

doi:10.1016/0146-6402(89)90027-1

Krohne, H. W. (1993). Vigilance and cognitive avoidance as concepts in coping research. In H. W. Krohne (Ed.), Attention and avoidance. Toronto: Hogrefe \& Huber.

Lachance, S., Ladouceur, R., \& Dugas, M. J. (1999). Elements explaining the tendency to worry. Applied Psychology-An International Review, 48, 187-196. doi:10.1080/026999499377600

Ladouceur, R., Gosselin, P., \& Dugas., M. J. (2000). Experimental manipulation of intolerance of uncertainty: A study of a theoretical model of worry. Behaviour Therapy and Research, 38, 933-941. doi:10.1016/S0005-7967(99)00133-3

Lal, M., \& Hassel, L. (1998). The joint impact of environmental uncertainty and tolerance of ambiguity on top managers' perceptions of the usefulness of non-conventional management accounting information. Scandinavian Journal of Management, 14, 259-271. doi:10.1016/S0956-5221(98)80010-9

Lange, R., \& Houran, J. (1998). Delusions of the paranormal: A haunting question of perception. Journal of Nervous and Mental Disease, 186, 637-645. doi:10.1097/00005053-199810000-00008

Lange, R., \& Houran, J. (1999). The role of fear in delusions of the paranormal. Journal of Nervous and Mental Disease, 187, 159-166. doi:10.1097/00005053-199903000-00005

Lauriola, M., \& Levin, I. P. (2001). Relating individual differences in Attitude toward Ambiguity to risky choices. Journal of Behavioural Decision Making, 14, 107-122. doi:10.1002/bdm.368

Lauriola, M., Levin, I. P., \& Hart, S. S. (2007). Common and distinct factors in decision making under ambiguity and risk: A psychometric study of individual differences. Organizational Behavior and Human Decision Processes, 104, 130-149. DOI:10.1016/J.OBHDP.2007.04.001

Le, A., Haller, C. S., Langer, E. J., \& Courvoisier, D. S. (2012). Mindful multitasking: The relationship between mindful flexibility and media multitasking. Computers in Human Behavior, 28, 1526-1532. doi:10.1016/j.chb.2012.03.022

Leong, C. H., \& Ward, C. (2000). Identity conflict in sojourners. International Journal of Intercultural Relations, 24, 763-776. doi:10.1016/S0147-1767(00)00030-4

Litman, J. A. (2010). Relationships between measures of I- and D-type curiosity, ambiguity tolerance, and need for closure: An initial test of the wanting-liking model of information-seeking. Personality and Individual Differences, 48, 397-402. doi:10.1016/j.paid.2009.11.005 


\section{A. FURNHAM, J. MARKS}

Lorsch, J. W., \& Morse, J. J. (1974). Organisations and their members. New York: Harper and Row.

MacDonald, A. P. (1970). Revised scale for ambiguity tolerance: Reliability and validity. Psychological Reports, 26, 791-798. doi:10.2466/pr0.1970.26.3.791

Marginson, D., \& Ogden, S. (2005). Coping with ambiguity through the budget: The positive effects of budgetary targets on managers' budgeting behaviours. Accounting, Organizations and Society, 30, 435456. doi:10.1016/j.aos.2004.05.004

McCrae, R. R. (1996). Social consequences of experiential openness. Psychological Bulletin, 120, 323-337. doi:10.1037/0033-2909.120.3.323

McLain, D. L. (1993). The MSTAT-I: A new measure of an individual's tolerance for ambiguity. Educational and Psychological Measurement, 53, 183-189. doi:10.1177/0013164493053001020

McLain, D. L. (2009). Evidence of the properties of an ambiguity tolerance measure: The multiple stimulus types ambiguity tolerance scale-II. Psychological Reports, 105, 975-988. doi:10.2466/pr0.105.3.975-988

McMullen, J. S., \& Shepherd, D. A. ( 2006). Entrepreneurial action and the role of uncertainty in the theory of the entrepreneur. Academy of Management Review, 31, 132-152. doi:10.5465/AMR.2006.19379628

Merrotsky, P. (2013). Tolerance of ambuity: A trait of the creative personality. Creativity Research Journal, 25, 232-237. doi:10.1080/10400419.2013.783762

Million, T. (1957). Authoritarianism, intolerance of ambiguity and rigidity under ego- and task-involving conditions. Journal of Abnormal and Social Psychology, 99, 29-33. doi:10.1037/h0040352

Morton, K. R., Worthley, J. S., Nitch, S. R., Lamberton, H. H., Loo, L. K., \& Testerman, J. K. (2000). Integration of cognition and emotion: A postformal operations model of physician-patient interaction. Journal of Adult Development, 7, 151-160. doi:10.1023/A:1009542229631

Neill, S., \& Rose, G. M. (2007). Achieving adaptive ends through equivocality: A study of organizational antecedents and consequences. Journal of Business Research, 60, 305-313. doi:10.1016/j.jbusres.2006.10.020

Norton, R. W. (1975). Measure of ambiguity tolerance. Journal of Personality Assessment, 39, 607-619. doi:10.1207/s15327752jpa3906 11

O'Connor, P. (1952). Ethnocentrism, "intolerance of ambiguity", and abstract reasoning ability. Journal of Abnormal and Social Psychology, 47, 526-530. doi: $10.1037 / \mathrm{h} 0056142$

Pillis, E., \& Reardon, K. (2007). The influence of personality traits and persuasive messages on entrepreneurial intention. Career Development International, 12, 382-396. doi:10.1108/13620430710756762

Rajagopal, L., \& Hamouz, F. L. (2009). Use of food attitudes and behaviors in determination of the personality characteristic of openness: A pilot study. International Journal of Intercultural Relations, 33, 254-258. doi:10.1016/j.ijintrel.2009.02.004

Rasch, G. (1960). Probabilistic models for some intelligence and attainment tests. Chicago, IL: MESA Press.

Richardson, P. S., Jain, A. K., \& Dick, A. (1996). Household store brand proneness: A framework. Journal of Retailing, 72, 159-185. doi:10.1016/S0022-4359(96)90012-3

Rong, G., \& Grover, V. (2009). Keeping up-to-date with information technology: Testing a model of technological knowledge renewal effectiveness for IT professionals. Information and Management, 46, 376-387. doi:10.1016/j.im.2009.07.002

Rush, M. C., Schoel, W. A., \& Barnard, S. M. (1995). Psychological resiliency in the public sector: "Hardiness" and pressure for change. Journal of Vocational Behavior, 46, 17-39. doi:10.1006/jvbe. 1995.1002

Rydell, S. (1966). Tolerance of ambiguity and semantic differential ratings. Psychological Reports, 19, 1303-1312. doi:10.2466/pr0.1966.19.3f.1303

Rydell, S. T., \& Rosen, E. (1966). Measurement and some correlates of need-cognition. Psychological Reports, 19, 139-165. doi:10.2466/pr0.1966.19.1.139

Saunders, D. (1955). Some preliminary interpretive material for the PRI research memorandum SS. Educational Testing Service.

Scott, W. A. (1966). Brief report: Measures of cognitive structure. Multivariate Behavioral Research, 1, 391-395. doi:10.1207/s15327906mbr0103 9

Scott, W. A. (1969). Structure of natural cognitions. Journal of Personality and Social Psychology, 12, 261-278. doi:10.1037/h0027734

Scott, W. A., Osgood, D. W., \& Peterson, C. (1979). Cognitive structure: Theory and measurement of individual differences. Washington: Winston \& Sons.

Steginga, S. K., \& Occhipinti, S. (2004). The application of the heuristic-systematic processing model to treatment decision-making about prostate cancer. Medical Decision Making, 24, 573-583. doi:10.1177/0272989X04271044

Stewart Jr., W. H., Carland, J. C., Carland, J. W., Watson, W. E., \& Sweo, R. (2003). Entrepreneurial dispositions and goal orientations: A comparative exploration of United States and Russian entrepreneurs. Journal of Small Business Management, 41, 27-46. doi:10.1111/1540-627X.00065

Storey, R. G., \& Aldag, R. J. (1983). Perceived environmental uncertainty: A test of an integrated explanatory model. Paper presented at the 43rd Annual meeting of the National Academy of Management.

Swami, V., Stieger, S., Pietschnig, J., \& Voracek, M. (2010). The disinterested play of thought: Individual differences and preference for surrealist motion pictures. Personality and Individual Differences, 48, 855-859. doi:10.1016/i.paid.2010.02.013

Tapanes, M. A., Smith, G. G., \& White, J. A. (2009). Cultural diversity in online learning: A study of the perceived effects of dissonance in levels of individualism/collectivism and tolerance of ambiguity. Internet and Higher Education, 12, 26-34. doi:10.1016/j.iheduc.2008.12.001

Teoh, H. Y., \& Foo, S. L. (1997). Moderating effects of tolerance for ambiguity and risk-taking propensity on the role conflict-perceived performance relationship: Evidence from Singaporean entrepreneurs. Journal of Business Venturing, 12, 67-81.

doi:10.1016/S0883-9026(96)00035-3

Thalbourne, M. A., \& Houran, J. (2000). Transliminality, the mental experience inventory and tolerance of ambiguity. Personality and Individual Differences, 28, 853-863. doi:10.1016/S0191-8869(99)00143-9

Thalbourne, M. A. (1994). Belief in the paranormal and its relationship to schizophrenia-relevant measures: A confirmatory study. British Journal of Clinical Psychology, 33, 78-80. doi:10.1111/i.2044-8260.1994.tb01097.x

Thalbourne, M. A., Dunbar, K. A., \& Delin, P. S. (1995). An investigation into correlates of belief in the paranormal. Journal of the American Society for Psychical Research, 89, 215-231.

Van Hook, C. W., \& Steele, C. (2002). Individual personality characteristics related to suggestibility. Psychological Reports, 91, 10071010. doi:10.2466/PR0.91.7.1007-1010

Weisbrod, E. (2009). The role of affect and tolerance of ambiguity in ethical decision making. Advances in Accounting, Incorporating Advances in International Accounting, 25, 57-63.

Westerberg, M., Singh, J., \& Hackner, E. (1997). Does the CEO matter? Scandanavian Journal of Management, 13, 251-270. doi:10.1016/S0956-5221(97)00011-0

Wittenberg, K. J., \& Norcross, J. C. (2001). Practitioner perfectionism: Relationship to ambiguity tolerance and work satisfaction. Journal of Clinical Psychology, 57, 1543-1550. doi:10.1002/jclp.1116

Wolfradt, U., \& Dorsh, S. (1995). Schizotypy and paranormal beliefs. A cross-cultural study between German and Mongolian pupils. In R. V. Quekelberghe (Ed.), Ethnopsychology and psychotherapy. Shamanistic healing rituals and modern therapies in comparison (pp. 228-245).

Wolfradt, U., \& Rademacher, J. (1999). Interpersonal intolerance of ambiguity as a clinical differential criterium. Zeitschrift for Differentielle und Diagnostiche Psychologie, 20, 10-20.

Wolfradt, U., Oubaid, V., Straube, E. R., Bischoff, N., \& Mischo, J. (1999). Thinking styles, schizotypal traits and anomalous experiences. Personality and Individual Differences, 27, 821-830. doi:10.1016/S0191-8869(99)00031-8

Yurtsever, G. (2001). Tolerance of ambiguity, information, and nego- 


\section{A. FURNHAM, J. MARKS}

tiation. Psychological Reports, 89, 57-64.

Yurtsever, G. (2008). Negotiators' profit predicted by cognitive reappraisal, suppression of emotions, misrepresentation of information, and tolerance of ambiguity. Perceptual and Motor Skills, 106, 590608. doi:10.2466/pms.106.2.590-608 